\title{
Dynamics of slowly varying fields in bianisotropic media
}

\author{
Stanislav I. Maslovski \\ Instituto de Telecomunicações, DEEC FCTUC Polo II - Pinhal de Marrocos \\ 3030-290 Coimbra, Portugal
}

\begin{abstract}
A theoretical framework for the quasi-monochromatic electromagnetic (EM) processes such as excitation and propagation of long wave packets in dispersive, dissipative, bianisotropic media with weak and slow nonlinearity is developed. The time-dependent EM fields associated with such processes are expressed as products of two functions: the slowly varying complex amplitude (SVCA) and the quickly oscillating carrier. The material parameters are treated as operators acting on time-dependent EM fields. By expanding these operators in the Maxwell equations in a series with respect to a small time scale parameter a system of equations for the SVCAs of the EM fields in such media is formulated. In the linear case, the dynamic equations for the SVCAs that correspond to the transverse components of the electric and magnetic fields resemble the vector transmission line equations. The obtained system of equations is used to derive the dyadic Green functions for the SVCAs of the EM fields in bianisotropic media. This framework is applied for modeling propagation of partially coherent EM radiation in a material whose parameters may depend on the amount of the EM energy that has passed through it. The same framework can be used in studying propagation of modulated EM waves through a waveguiding system that includes bianisotropic metamaterial components.
\end{abstract}

Keywords: bianisotropic media, metamaterials, slowly varying amplitude method, slow nonlinearity, wave packet, dispersive media, Green function

\section{INTRODUCTION}

In this paper we develop a theoretical framework for quasi-monochromatic electromagnetic processes in dispersive media such as propagation of long wave packets. The application of this approach is the area of super-Planckian radiative heat transfer in metamaterials. ${ }^{1-6}$ In the processes that we consider, most of the spectral energy is concentrated in a short interval around a single frequency: $\omega_{0}-\Delta \omega / 2 \leq \omega \leq \omega_{0}+\Delta \omega / 2, \Delta \omega \ll \omega_{0}$. Respectively,

Further author information: (Send correspondence to S.I.M.)

S.I.M.: E-mail: stas@co.it.pt 
the time-dependent EM fields associated with such processes can be expressed as products of two functions: the slowly varying complex amplitude (SVCA) and the quickly oscillating carrier. Because the characteristic time scales of the two functions may differ by many orders of magnitude, the slowly non-linear and dispersive response of the medium relates only to the SVCA. Thus, the time-dependent electric and magnetic field vectors $\mathbf{F} \equiv \mathbf{E}, \mathbf{H}$ can be written in our case as

$$
\mathbf{F}(t)=\frac{1}{2 \pi} \int_{\omega_{0}-\Delta \omega / 2}^{\omega_{0}+\Delta \omega / 2} \mathbf{F}_{\omega}(\omega) e^{-i \omega t} d \omega+\text { c.c. }=\frac{1}{2} \mathbf{F}_{\mathrm{m}}(t) e^{-i \omega_{0} t}+\text { c.c. },
$$

where "c.c." denotes the complex conjugate of the previous term. The quantity

$$
\mathbf{F}_{\mathrm{m}}(t)=\frac{1}{\pi} \int_{-\Delta \omega / 2}^{+\Delta \omega / 2} \mathbf{F}_{\omega}\left(\omega_{0}+\Omega\right) e^{-i \Omega t} d \Omega
$$

is the SVCA (the time envelope) of the process. Note that for monochromatic processes with delta-functional spectral density, Eqs. (1) and (2) imply that $\mathbf{F}_{\mathrm{m}}$ is constant and its magnitude simply equals the oscillating field amplitude.

From the above definition it follows that for any quantity $\mathbf{G}(t)=\overline{\bar{\alpha}} \cdot \mathbf{F}(t)$, where $\overline{\bar{\alpha}}$ is a dyadic linear integrodifferential operator with the Fourier transform $\overline{\bar{\alpha}}_{\omega}=\left.\overline{\bar{\alpha}}_{\omega}\right|_{\omega_{0}}+\left.\left(\omega-\omega_{0}\right)\left(\partial \overline{\bar{\alpha}}_{\omega} / \partial \omega\right)\right|_{\omega_{0}}+\cdots$, the SVCA G $\mathbf{m}_{\mathrm{m}}(t)$ of the quantity $\mathbf{G}(t)$ can be expanded as

$$
\mathbf{G}_{\mathrm{m}}(t)=\left.\overline{\bar{\alpha}}_{\omega}\right|_{\omega_{0}} \cdot \mathbf{F}_{\mathrm{m}}(t)+\left.i \frac{\partial \overline{\bar{\alpha}}_{\omega}}{\partial \omega}\right|_{\omega_{0}} \cdot \frac{\partial \mathbf{F}_{\mathrm{m}}(t)}{\partial t}+\cdots
$$

and the SVCA of the time derivative of the same quantity can be expanded as

$$
\left[\frac{\partial \mathbf{G}(t)}{\partial t}\right]_{\mathrm{m}}=-\left.i \omega_{0} \overline{\bar{\alpha}}_{\omega}\right|_{\omega_{0}} \cdot \mathbf{F}_{\mathrm{m}}(t)+\left.\frac{\partial\left(\omega \overline{\bar{\alpha}}_{\omega}\right)}{\partial \omega}\right|_{\omega_{0}} \cdot \frac{\partial \mathbf{F}_{\mathrm{m}}(t)}{\partial t}+\cdots
$$

In order to account for slow and weak nonlinearity, we assume that $\overline{\bar{\alpha}}_{\omega}$, which can be, e.g., dielectric permittivity dyadic at a given point, is a function of a time-dependent parameter $T$ (e.g., local temperature), which in turn depends on the amount of work the EM field has performed on the material in the vicinity of the selected point. For instance, if only the work due to the material loss accounted by $\overline{\bar{\alpha}}_{\omega}^{\prime \prime}=\operatorname{Im}(\overline{\bar{\alpha}})^{*}$ is taken into account, then, at the selected point,

$$
T(t)=T(0)+\frac{1}{2 c_{T}} \int_{0}^{t} d \tau \mathbf{F}_{m}^{\dagger}(\tau) \cdot \overline{\bar{\alpha}}_{\omega}^{\prime \prime}(T(\tau)) \cdot \mathbf{F}_{m}(\tau),
$$

where $c_{T}$ is a parameter that has the meaning of the thermal capacity of the material per unit volume. Such nonlinearity is considered weak and slow when $c_{T}$ is enough large, so that

$$
\frac{1}{T} \frac{d T}{d t} \ll \frac{1}{\left|\mathbf{F}_{m}\right|} \frac{d\left|\mathbf{F}_{m}\right|}{d t}
$$

\footnotetext{
${ }^{*}$ Here, $\operatorname{Im}(\overline{\bar{\alpha}})=\left(\overline{\bar{\alpha}}-\overline{\bar{\alpha}}^{\dagger}\right) /(2 i)$.
} 
which ensures applicability of expansions (3) and (4). Therefore, when considering propagation of elementary excitations (certain wave packets, more details ahead) with characteristic duration $\tau_{p} \sim 2 \pi / \Delta \omega$, we may neglect the change in $T$ (and, respectively, $\overline{\bar{\alpha}}_{\omega}$ ) during time intervals $\Delta t \sim \tau_{p}$, so that the material can be considered effectively linear with respect to the propagation of such a single pulse. However, propagation of a longer excitation represented by a series of such pulses, will be influenced by the gradual change in $T$ caused by every passing elementary pulse. Thus, the slow and weak nonlinearity that we consider works as an accumulative memory mechanism.

In what follows we derive equations which describe the dynamics of such SVCAs of the electric and magnetic fields in dispersive uniaxial bianisotropic media with such weak and slow nonlinearity.

\section{MAXWELL EQUATIONS FOR SLOWLY VARYING FIELD AMPLITUDES}

In order to consider propagation of the elementary excitations mentioned in the previous section, we consider the macroscopic Maxwell equations in presence of both electric and magnetic sources ${ }^{\dagger}$, which can be written as

$$
\frac{\partial \mathbf{D}}{\partial t}=\nabla \times \mathbf{H}-\mathbf{J}, \quad \frac{\partial \mathbf{B}}{\partial t}=-\nabla \times \mathbf{E}-\mathbf{K},
$$

with the electric and the magnetic current densities $\mathbf{J}$ and $\mathbf{K}$ satisfying the continuity equations

$$
\nabla \cdot \mathbf{J}=-\frac{\partial \rho}{\partial t}, \quad \nabla \cdot \mathbf{K}=-\frac{\partial \kappa}{\partial t}
$$

where $\rho$ and $\kappa$ are the electric and magnetic charge densities, respectively.

The electric displacement $\mathbf{D}$ and the magnetic induction $\mathbf{B}$ in bianisotropic media satisfy the following constitutive relations, written in a matrix form:

$$
\left(\begin{array}{l}
\mathbf{D} \\
\mathbf{B}
\end{array}\right)=\left(\begin{array}{cc}
\overline{\bar{\varepsilon}} & \overline{\bar{\xi}} \\
\overline{\bar{\zeta}} & \overline{\bar{\mu}}
\end{array}\right) \cdot\left(\begin{array}{l}
\mathbf{E} \\
\mathbf{H}
\end{array}\right)=\mathfrak{M} \cdot\left(\begin{array}{l}
\mathbf{E} \\
\mathbf{H}
\end{array}\right) .
$$

In this relation, the components of the material matrix $\mathfrak{M}$ are dyadic integro-differential operators ${ }^{\ddagger}$ corresponding to the constitutive parameters of the medium: the effective permittivity $\overline{\bar{\varepsilon}}$, the effective permeability $\overline{\bar{\mu}}$, and the effective electro-magnetic and magneto-electric coupling dyadics $\overline{\bar{\xi}}$ and $\overline{\bar{\zeta}}$. By substituting Eq. (9) into the Maxwell equations (7) we obtain the Maxwell equations in the so-called 6-vector form:

$$
\frac{\partial}{\partial t}\left(\mathfrak{M} \cdot\left(\begin{array}{l}
\mathbf{E} \\
\mathbf{H}
\end{array}\right)\right)=\left(\begin{array}{cc}
0 & \nabla \times \overline{\overline{\mathrm{I}}} \\
-\nabla \times \overline{\overline{\mathrm{I}}} & 0
\end{array}\right) \cdot\left(\begin{array}{l}
\mathbf{E} \\
\mathbf{H}
\end{array}\right)-\left(\begin{array}{l}
\mathbf{J} \\
\mathbf{K}
\end{array}\right) .
$$

\footnotetext{
${ }^{\dagger}$ The effective magnetic source current density $\mathbf{K}$ can be understood as resulting from the divergence-free, solenoidal electric currents.

${ }^{\ddagger}$ We understand these quantities as operators acting on the fields as functions of time, in order to take into account the material dispersion, and the parameter $T$, in order to account for the slow and weak nonlinearity.
} 
In the frequency domain, the material relations (9) can be written as

$$
\left(\begin{array}{c}
\mathbf{D}_{\omega} \\
\mathbf{B}_{\omega}
\end{array}\right)=\left(\begin{array}{cc}
\overline{\bar{\varepsilon}}_{\omega} & \overline{\bar{\xi}}_{\omega} \\
\overline{\bar{\zeta}}_{\omega} & \overline{\bar{\mu}}_{\omega}
\end{array}\right) \cdot\left(\begin{array}{c}
\mathbf{E}_{\omega} \\
\mathbf{H}_{\omega}
\end{array}\right)=\mathfrak{M}_{\omega} \cdot\left(\begin{array}{c}
\mathbf{E}_{\omega} \\
\mathbf{H}_{\omega}
\end{array}\right) \text {. }
$$

When interested in the quasi-monochromatic processes described by the slow varying field amplitudes introduced in Sec. 1, we can expand the Fourier-transformed material matrix $\mathfrak{M}_{\omega}$ in a Taylor series in the vicinity of the frequency $\omega_{0}$ :

$$
\mathfrak{M}_{\omega}=\left.\mathfrak{M}_{\omega}\right|_{\omega_{0}}+\left.\Omega \frac{\partial \mathfrak{M}_{\omega}}{\partial \omega}\right|_{\omega_{0}}+\cdots
$$

where $\Omega=\omega-\omega_{0}$, and drop the higher-order terms starting with $\Omega^{2}$. Then, by using the results of Sec. 1 , we find that the slowly varying complex amplitudes $\mathbf{D}_{\mathrm{m}}(t)$ and $\mathbf{B}_{\mathrm{m}}(t)$ satisfy

$$
\left.\left(\begin{array}{l}
\mathbf{D}_{\mathrm{m}} \\
\mathbf{B}_{\mathrm{m}}
\end{array}\right) \approx \mathfrak{M}_{\omega}\right|_{\omega_{0}} \cdot\left(\begin{array}{l}
\mathbf{E}_{\mathrm{m}} \\
\mathbf{H}_{\mathrm{m}}
\end{array}\right)+\left.i \frac{\partial \mathfrak{M}_{\omega}}{\partial \omega}\right|_{\omega_{0}} \cdot \frac{\partial}{\partial t}\left(\begin{array}{c}
\mathbf{E}_{\mathrm{m}} \\
\mathbf{H}_{\mathrm{m}}
\end{array}\right)
$$

Therefore, the slowly varying amplitudes of the time derivatives of the $\mathbf{D}$ and $\mathbf{B}$ vectors can be now written as

$$
\left[\frac{\partial}{\partial t}\left(\begin{array}{l}
\mathbf{D} \\
\mathbf{B}
\end{array}\right)\right]_{\mathrm{m}} \approx-\left.i \omega_{0} \mathfrak{M}_{\omega}\right|_{\omega_{0}}\left(\begin{array}{l}
\mathbf{E}_{\mathrm{m}} \\
\mathbf{H}_{\mathrm{m}}
\end{array}\right)+\left.\frac{\partial\left(\omega \mathfrak{M}_{\omega}\right)}{\partial \omega}\right|_{\omega_{0}} \cdot \frac{\partial}{\partial t}\left(\begin{array}{l}
\mathbf{E}_{\mathrm{m}} \\
\mathbf{H}_{\mathrm{m}}
\end{array}\right) .
$$

Note that the second derivatives of $\mathbf{E}_{\mathrm{m}}$ and $\mathbf{H}_{\mathrm{m}}$ with respect to time do not enter Eq. (14), in agreement with the accuracy of the expansion (12).

Based on the above results, we can now write the Maxwell equations for the slowly varying amplitudes of the electromagnetic fields, by replacing the corresponding terms in Eq. (10) with their expressions in terms of the slowly varying envelops. The result is

$$
\left.\frac{\partial\left(\omega \mathfrak{M}_{\omega}\right)}{\partial \omega}\right|_{\omega_{0}} \cdot \frac{\partial}{\partial t}\left(\begin{array}{c}
\mathbf{E}_{\mathrm{m}} \\
\mathbf{H}_{\mathrm{m}}
\end{array}\right)=\left(\left.i \omega_{0} \mathfrak{M}_{\omega}\right|_{\omega_{0}}+\left(\begin{array}{cc}
0 & \nabla \times \overline{\overline{\mathrm{I}}} \\
-\nabla \times \overline{\overline{\mathrm{I}}} & 0
\end{array}\right)\right) \cdot\left(\begin{array}{c}
\mathbf{E}_{\mathrm{m}} \\
\mathbf{H}_{\mathrm{m}}
\end{array}\right)-\left(\begin{array}{c}
\mathbf{J}_{\mathrm{m}} \\
\mathbf{K}_{\mathrm{m}}
\end{array}\right),
$$

where $\overline{\overline{\mathrm{I}}}$ is the identity dyadic, and $\mathbf{J}_{\mathrm{m}}$ and $\mathbf{K}_{\mathrm{m}}$ are the slowly varying amplitudes of the electric and magnetic current densities, respectively.

It can be shown that in passive dispersive media with low dissipation, the matrix $\partial\left(\omega \mathfrak{M}_{\omega}\right) / \partial \omega$ is a positive definite matrix and is, therefore, non-singular. In general, in all cases when the mentioned matrix is non-singular we can rewrite Eq. (15) as

$$
\frac{\partial}{\partial t}\left(\begin{array}{l}
\mathbf{E}_{\mathrm{m}} \\
\mathbf{H}_{\mathrm{m}}
\end{array}\right)=\left[\frac{\partial\left(\omega \mathfrak{M}_{\omega}\right)}{\partial \omega}\right]_{\omega_{0}}^{-1} \cdot\left\{\left(\left.i \omega_{0} \mathfrak{M}_{\omega}\right|_{\omega_{0}}+\left(\begin{array}{cc}
0 & \nabla \times \overline{\bar{I}} \\
-\nabla \times \overline{\overline{\mathrm{I}}} & 0
\end{array}\right)\right) \cdot\left(\begin{array}{l}
\mathbf{E}_{\mathrm{m}} \\
\mathbf{H}_{\mathrm{m}}
\end{array}\right)-\left(\begin{array}{c}
\mathbf{J}_{\mathrm{m}} \\
\mathbf{K}_{\mathrm{m}}
\end{array}\right)\right\} .
$$

In what follows, we restrict our consideration only by such cases when Eq. (16) holds. Note that this form of Eq. (16) in which the time derivatives of the slowly varying amplitudes are expressed through the spatial derivatives of the same quantities allows, for instance, for a direct implementation of a numerical solver. 


\section{VECTOR TELEGRAPHER'S EQUATION FOR WAVE PACKETS IN DISPERSIVE UNIAXIAL BIANISOTROPIC MEDIA}

An important special case of anisotropic media is when there exist a single selected direction such that the electromagnetic response of the medium along this direction is different from the response to the field components orthogonal to the same direction. This is the case of uniaxial bianisotropic materials, in which the constitutive parameters assume the form

$$
\overline{\bar{\varepsilon}}_{\omega}=\overline{\bar{\varepsilon}}_{\mathrm{t}}+\varepsilon_{\mathrm{n}} \hat{\mathbf{z}} \otimes \hat{\mathbf{z}}, \quad \overline{\bar{\mu}}_{\omega}=\overline{\bar{\mu}}_{\mathrm{t}}+\mu_{\mathrm{n}} \hat{\mathbf{z}} \otimes \hat{\mathbf{z}}, \quad \overline{\bar{\xi}}_{\omega}=\overline{\bar{\xi}}_{\mathrm{t}}+\xi_{\mathrm{n}} \hat{\mathbf{z}} \otimes \hat{\mathbf{z}}, \quad \overline{\bar{\zeta}}_{\omega}=\overline{\bar{\zeta}}_{\mathrm{t}}+\zeta_{\mathrm{n}} \hat{\mathbf{z}} \otimes \hat{\mathbf{z}},
$$

where $\hat{\mathbf{z}}$ is the unit vector along the main anisotropy axis (which we select coincident with the $z$-axis direction) and the subindex $(\ldots)_{t}$ denotes the dyadic components in the transverse plane orthogonal to $\hat{\mathbf{z}}$. These dyadics satisfy $(\hat{\mathbf{z}} \otimes \hat{\mathbf{z}})_{\times}^{\times} \overline{\bar{\varepsilon}}_{\mathrm{t}}=\overline{\bar{\varepsilon}}_{\mathrm{t}},(\hat{\mathbf{z}} \otimes \hat{\mathbf{z}})_{\times}^{\times} \overline{\bar{\mu}}_{\mathrm{t}}=\overline{\bar{\mu}}_{\mathrm{t}},(\hat{\mathbf{z}} \otimes \hat{\mathbf{z}}){ }_{\times}^{\times} \overline{\bar{\xi}}_{\mathrm{t}}=\overline{\bar{\xi}}_{\mathrm{t}},(\hat{\mathbf{z}} \otimes \hat{\mathbf{z}}){ }_{\times}^{\times} \overline{\bar{\zeta}}_{\mathrm{t}}=\overline{\bar{\zeta}}_{\mathrm{t}}$, where $(\mathbf{a} \otimes \mathbf{b})_{\times}^{\times}(\mathbf{c} \otimes \mathbf{d}) \equiv(\mathbf{a} \times \mathbf{c}) \otimes(\mathbf{b} \times \mathbf{d})$. In the reciprocal uniaxial bianisotropic media, the transverse dyadics $\overline{\bar{\varepsilon}}_{\mathrm{t}}$ and $\overline{\bar{\mu}}_{\mathrm{t}}$ reduce to $\overline{\bar{\varepsilon}}_{\mathrm{t}}=\varepsilon_{\mathrm{t}} \overline{\overline{\mathrm{I}}}_{\mathrm{t}}, \overline{\bar{\mu}}_{\mathrm{t}}=\mu_{\mathrm{t}} \overline{\overline{\mathrm{I}}}_{\mathrm{t}}$, where $\overline{\overline{\mathrm{I}}}_{\mathrm{t}}=\overline{\overline{\mathrm{I}}}-\hat{\mathbf{z}} \otimes \hat{\mathbf{z}}$, and the dyadics $\overline{\bar{\xi}}_{\omega}$ and $\overline{\bar{\zeta}}_{\omega}$ are such that $\overline{\bar{\xi}}_{\omega}=-\overline{\bar{\zeta}}_{\omega}^{T}$, where $(\ldots)^{T}$ denotes the dyadic transpose operation. In the general uniaxial bianisotropic media, $\overline{\bar{\varepsilon}}_{\mathrm{t}}$ and $\overline{\bar{\mu}}_{\mathrm{t}}$ may have an additional antisymmetric component and $\overline{\bar{\xi}}_{\omega}$ and $\overline{\bar{\zeta}}_{\omega}$ may be such that $\overline{\bar{\zeta}}_{\omega} \neq-\overline{\bar{\zeta}}_{\omega}^{T}$.

Decomposition (17) allows one to rewrite the Maxwell equations in the uniaxial bianisotropic media in such a way that the $z$-components of the fields are eliminated. Namely, when working in the frequency domain, after some dyadic algebra the following result can be obtained:

$$
-i \omega \tilde{\mathfrak{M}}_{\omega}\left(\nabla_{\mathrm{t}}\right) \cdot\left(\begin{array}{c}
\mathbf{E}_{\omega, \mathrm{t}} \\
\mathbf{H}_{\omega, \mathrm{t}}
\end{array}\right)=\mathfrak{J} \frac{\partial}{\partial z} \cdot\left(\begin{array}{c}
\mathbf{E}_{\omega, \mathrm{t}} \\
\mathbf{H}_{\omega, \mathrm{t}}
\end{array}\right)-\mathfrak{S}_{\omega}\left(\nabla_{\mathrm{t}}\right) \cdot\left(\begin{array}{c}
\mathbf{J}_{\omega} \\
\mathbf{K}_{\omega}
\end{array}\right)
$$

where $\nabla_{\mathrm{t}}=\overline{\overline{\mathrm{I}}}_{\mathrm{t}} \cdot \nabla, \mathbf{E}_{\omega, \mathrm{t}}=\overline{\overline{\mathrm{I}}}_{\mathrm{t}} \cdot \mathbf{E}_{\omega}, \mathbf{H}_{\omega, \mathrm{t}}=\overline{\overline{\mathrm{I}}}_{\mathrm{t}} \cdot \mathbf{H}_{\omega}, \mathfrak{J}=\left(\begin{array}{cc}0 & \hat{\mathbf{z}} \times \overline{\overline{\mathrm{I}}}_{\mathrm{t}} \\ -\hat{\mathbf{z}} \times \overline{\overline{\mathrm{I}}}_{\mathrm{t}} & 0\end{array}\right)$, and

$$
\begin{aligned}
& \tilde{\mathfrak{M}}_{\omega}\left(\nabla_{\mathrm{t}}\right)=\left(\begin{array}{cc}
\overline{\bar{\varepsilon}}_{\mathrm{t}} & \overline{\bar{\xi}}_{\mathrm{t}} \\
\overline{\bar{\zeta}}_{\mathrm{t}} & \overline{\bar{\mu}}_{\mathrm{t}}
\end{array}\right)+\frac{1}{\omega^{2}} \mathfrak{J} \cdot \nabla_{\mathrm{t}}\left(\begin{array}{cc}
\varepsilon_{\mathrm{n}} & \xi_{\mathrm{n}} \\
\zeta_{\mathrm{n}} & \mu_{\mathrm{n}}
\end{array}\right)^{-1} \otimes \nabla_{\mathrm{t}} \cdot \mathfrak{J}, \\
& \mathfrak{S}_{\omega}\left(\nabla_{\mathrm{t}}\right)=\left(\begin{array}{cc}
\overline{\overline{\mathrm{I}}}_{\mathrm{t}} & 0 \\
0 & \overline{\overline{\mathrm{I}}}_{\mathrm{t}}
\end{array}\right)+\frac{1}{i \omega} \mathfrak{J} \cdot \nabla_{\mathrm{t}}\left(\begin{array}{cc}
\varepsilon_{\mathrm{n}} & \xi_{\mathrm{n}} \\
\zeta_{\mathrm{n}} & \mu_{\mathrm{n}}
\end{array}\right)^{-1} \otimes \hat{\mathbf{z}} .
\end{aligned}
$$

The dynamic equations for the slowly varying amplitudes of the corresponding quantities are immediately obtained from Eq. (18), using the same procedure as for Eqs. (10) and (16):

$$
\frac{\partial}{\partial t}\left(\begin{array}{c}
\mathbf{E}_{\mathrm{m}, \mathrm{t}} \\
\mathbf{H}_{\mathrm{m}, \mathrm{t}}
\end{array}\right)=\left[\frac{\partial\left(\omega \tilde{\mathfrak{M}}_{\omega}\left(\nabla_{\mathrm{t}}\right)\right)}{\partial \omega}\right]_{\omega_{0}}^{-1} \cdot\left\{\left(\left.i \omega_{0} \tilde{\mathfrak{M}}_{\omega}\left(\nabla_{\mathrm{t}}\right)\right|_{\omega_{0}}+\mathfrak{J} \frac{\partial}{\partial z}\right) \cdot\left(\begin{array}{c}
\mathbf{E}_{\mathrm{m}, \mathrm{t}} \\
\mathbf{H}_{\mathrm{m}, \mathrm{t}}
\end{array}\right)-\left(\begin{array}{c}
\tilde{\mathbf{J}}_{\mathrm{m}, \mathrm{t}} \\
\tilde{\mathbf{K}}_{\mathrm{m}, \mathrm{t}}
\end{array}\right)\right\}
$$


where

$$
\left(\begin{array}{c}
\tilde{\mathbf{J}}_{\mathrm{m}, \mathrm{t}} \\
\tilde{\mathbf{K}}_{\mathrm{m}, \mathrm{t}}
\end{array}\right)=\left(\mathfrak{S}_{\omega}\left(\nabla_{\mathrm{t}}\right)+\left.i \frac{\partial \mathfrak{S}_{\omega}\left(\nabla_{\mathrm{t}}\right)}{\partial \omega}\right|_{\omega_{0}} \frac{\partial}{\partial t}\right) \cdot\left(\begin{array}{c}
\mathbf{J}_{\mathrm{m}} \\
\mathbf{K}_{\mathrm{m}}
\end{array}\right)
$$

Eq. (21) can be understood as the Maxwell equations (16) reformulated as for an effectively one-dimensional wave propagation along the $z$-axis. Indeed, in the case when the material parameters are uniform in the transverse plane, when taking a spatial Fourier transform of (21) in this plane the operator $\nabla_{\mathrm{t}}$ transforms as $\nabla_{\mathrm{t}} \mapsto i \mathbf{k}_{t}$, where $\mathbf{k}_{\mathrm{t}}$ is the transverse wave vector, and Eq. (21) immediately reduces to a system of two differential equations in $\partial / \partial t$ and $\partial / \partial z$.

We can rewrite Eq. (21) slightly in order to highlight this fact even further, using the fact that $\mathfrak{J} \cdot \mathfrak{J}=\left(\begin{array}{cc}\overline{\overline{\mathrm{I}}}_{\mathrm{t}} & 0 \\ 0 & \overline{\overline{\mathrm{I}}}_{\mathrm{t}}\end{array}\right)$. After multiplying Eq. (21) by $\mathfrak{J}$ and rearranging the terms we find that

$$
\frac{\partial}{\partial z}\left(\begin{array}{c}
\mathbf{E}_{\mathrm{m}, \mathrm{t}} \\
\mathbf{H}_{\mathrm{m}, \mathrm{t}}
\end{array}\right)=\left(i \mathfrak{K}\left(\omega_{0}, \nabla_{\mathrm{t}}\right)-\frac{\partial \mathfrak{K}\left(\omega_{0}, \nabla_{\mathrm{t}}\right)}{\partial \omega_{0}} \cdot \frac{\partial}{\partial t}\right) \cdot\left(\begin{array}{c}
\mathbf{E}_{\mathrm{m}, \mathrm{t}} \\
\mathbf{H}_{\mathrm{m}, \mathrm{t}}
\end{array}\right)+\mathfrak{J} \cdot\left(\begin{array}{c}
\tilde{\mathbf{J}}_{\mathrm{m}, \mathrm{t}} \\
\tilde{\mathbf{K}}_{\mathrm{m}, \mathrm{t}}
\end{array}\right),
$$

where $\mathfrak{K}\left(\omega_{0}, \nabla_{\mathrm{t}}\right)=-\left.\omega_{0} \mathfrak{J} \cdot \tilde{\mathfrak{M}}_{\omega}\left(\nabla_{\mathrm{t}}\right)\right|_{\omega_{0}}$. Eq. (23) can be called the vector telegrapher's equation for the SVCA of the electromagnetic field in bianisotropic media.

\section{SOLUTION OF VECTOR TELEGRAPHER'S EQUATION: ENVELOP GREEN FUNCTION}

First, let us consider the situation when the source term in Eq. (23) vanishes. In this case, the vector telegrapher's equation reads

$$
\frac{\partial \mathbf{F}}{\partial z}=\left(i \mathfrak{K}\left(\omega_{0}, \nabla_{\mathrm{t}}\right)-\frac{\partial \mathfrak{K}\left(\omega_{0}, \nabla_{\mathrm{t}}\right)}{\partial \omega_{0}} \frac{\partial}{\partial t}\right) \cdot \mathbf{F}
$$

where $\mathbf{F}=\left(\begin{array}{c}\mathbf{E}_{\mathrm{m}, \mathrm{t}} \\ \mathbf{H}_{\mathrm{m}, \mathrm{t}}\end{array}\right)$. This equation can be solved in the standard way, e.g. by separation of variables.

In order to find a more elegant way of resolving (24), let us consider a special case of Eq. (24) when the operators $\mathfrak{K}\left(\omega_{0}, \nabla_{\mathrm{t}}\right)$ and $\partial \mathfrak{K}\left(\omega_{0}, \nabla_{\mathrm{t}}\right) / \partial \omega_{0}$ commute $^{\S}$ and are independent of $z$. In this case the solution of Eq. (24) can be formally written as

$$
\begin{aligned}
\mathbf{F}(x, y, z, t) & =e^{z\left(i \mathfrak{K}\left(\omega_{0}, \nabla_{\mathrm{t}}\right)-\frac{\partial \mathfrak{K}\left(\omega_{0}, \nabla_{\mathrm{t}}\right)}{\partial \omega_{0}} \frac{\partial}{\partial t}\right)} \cdot \mathbf{F}_{0}(x, y, t) \\
& =e^{i z \mathfrak{K}\left(\omega_{0}, \nabla_{\mathrm{t}}\right)} \cdot e^{-z \frac{\partial \mathfrak{K}\left(\omega_{0}, \nabla_{\mathrm{t}}\right)}{\partial \omega_{0}} \frac{\partial}{\partial t}} \cdot \mathbf{F}_{0}(x, y, t),
\end{aligned}
$$

\footnotetext{
${ }^{\S}$ Which means that there exist a basis in which the two operators are diagonal.
} 
where $\mathbf{F}_{0}(x, y, t)$ is arbitrary. In particular, when $\mathbf{F}_{0}=e^{-i \Omega t} \mathbf{A}(x, y)$, where $\Omega \in[-\Delta \omega / 2 ;+\Delta \omega / 2]$ and $\mathbf{A}(x, y)$ is an eigenfunction of the operator $\mathfrak{K}\left(\omega_{0}, \nabla_{\mathrm{t}}\right)$ such that $\mathfrak{K}\left(\omega_{0}, \nabla_{\mathrm{t}}\right) \cdot \mathbf{A}=k_{z} \mathbf{A}$, we obtain from Eq. (25):

$$
\mathbf{F}_{\Omega, k_{z}}=e^{i k_{z} z} e^{-i \Omega\left(t-\frac{\partial k_{z}}{\partial \omega_{0}} z\right)} \mathbf{A}(x, y)
$$

from which it follows that, in the considered case, the solutions of Eq. (24) are wave packets with the polarization state and the field distribution in the transverse plane described by the eigenfunctions $\mathbf{A}(x, y)$, the fast spatial phase variation described by the term $e^{i k_{z} z}$, and with a slowly varying envelope function $f\left(t-\frac{\partial k_{z}}{\partial \omega_{0}} z\right)$ with finite spectrum fitting into the interval $[-\Delta \omega / 2 ;+\Delta \omega / 2]$. Such wave packets propagate along the $z$-axis with the speed $v_{\mathrm{g}}=\left(\partial k_{z} / \partial \omega_{0}\right)^{-1}$, which is an analog of the group velocity in the considered medium. Therefore, we can express the general solution $\mathbf{F}(x, y, z, t)$ in this case as

$$
\mathbf{F}(x, y, z, t)=\sum_{n} f_{n}\left(t-z / v_{g}^{(n)}\right) e^{i k_{z}^{(n)} z} \mathbf{A}^{(n)}(x, y),
$$

where the summation is taken over all linearly independent eigenfunctions $\mathbf{A}^{(n)}(x, y)$.

Let us now consider the vector telegrapher's equation with a source term under the same assumption that the operators $\mathfrak{K}\left(\omega_{0}, \nabla_{\mathrm{t}}\right)$ and $\partial \mathfrak{K}\left(\omega_{0}, \nabla_{\mathrm{t}}\right) / \partial \omega_{0}$ commute and do not depend on $z$. The equation that we need to solve reads

$$
\frac{\partial \mathbf{F}}{\partial z}=\left(i \mathfrak{K}\left(\omega_{0}, \nabla_{\mathrm{t}}\right)-\frac{\partial \mathfrak{K}\left(\omega_{0}, \nabla_{\mathrm{t}}\right)}{\partial \omega_{0}} \frac{\partial}{\partial t}\right) \cdot \mathbf{F}+\mathbf{S}_{0} e^{-i \Omega t} \delta\left(z-z_{0}\right),
$$

where the source term is set to a slowly oscillating function concentrated around the point $z=z_{0}$. Knowing the solution of the homogeneous equation, we may look for a particular solution of the inhomogeneous equation (28) in the following form:

$$
\begin{aligned}
\mathbf{F}_{s} & =\sum_{(+n)} C^{(+n)} \theta\left(z-z_{0}\right) e^{i k_{z}^{(+n)} z} e^{-i \Omega\left(t-\frac{\partial k_{z}^{(+n)}}{\partial \omega_{0}} z\right)} \mathbf{A}^{(+n)}(x, y) \\
& +\sum_{(-n)} C^{(-n)} \theta\left(z_{0}-z\right) e^{i k_{z}^{(-n)} z} e^{-i \Omega\left(t-\frac{\partial k_{z}^{(-n)}}{\partial \omega_{0}} z\right)} \mathbf{A}^{(-n)}(x, y),
\end{aligned}
$$

where $\theta(z)$ is the Heaviside step function, and the summations over $( \pm n)$ mean the summation over the components with the corresponding sign of the real part of the group delay factor $\partial k_{z} / \partial \omega_{0}$.

After substituting this into Eq. (28) and equating the coefficients at the delta-functional terms we obtain

$$
\sum_{(+n)} C^{(+n)} e^{i k_{z}^{(+n)} z_{0}} e^{i \Omega \frac{\partial k_{z}^{(+n)}}{\partial \omega_{0}} z_{0}} \mathbf{A}^{(+n)}-\sum_{(-n)} C^{(-n)} e^{i k_{z}^{(-n)} z_{0}} e^{i \Omega \frac{\partial k_{z}^{(-n)}}{\partial \omega_{0}} z_{0}} \mathbf{A}^{(-n)}=\mathbf{S}_{0} .
$$

Without any loss of generality, we may assume that the eigenfunctions $\mathbf{A}^{(n)}(x, y) \equiv\left|\mathbf{A}^{(n)}\right\rangle$ form a Riesz basis. Then, by introducing the reciprocal basis $\left\langle\tilde{\mathbf{A}}^{(n)}\right|$ such that the inner product satisfies

$$
\left\langle\tilde{\mathbf{A}}^{(m)} \mid \mathbf{A}^{(n)}\right\rangle=\delta_{m n},
$$


where $\delta_{m n}$ is Kronecker's delta, we find that

$$
C^{( \pm n)}= \pm e^{-i k_{z}^{( \pm n)} z_{0}} e^{-i \Omega \frac{\partial k_{z}^{( \pm n)}}{\partial \omega_{0}} z_{0}}\left\langle\tilde{\mathbf{A}}^{( \pm n)} \mid \mathbf{S}_{0}\right\rangle
$$

Respectively,

$$
\begin{aligned}
\mathbf{F}_{s} & =\sum_{(+n)} \theta\left(z-z_{0}\right) e^{i k_{z}^{(+n)}\left(z-z_{0}\right)} e^{-i \Omega\left(t-\frac{\partial k_{z}^{(+n)}}{\partial \omega_{0}}\left(z-z_{0}\right)\right)}\left|\mathbf{A}^{(+n)}\right\rangle\left\langle\tilde{\mathbf{A}}^{(+n)} \mid \mathbf{S}_{0}\right\rangle \\
& -\sum_{(-n)} \theta\left(z_{0}-z\right) e^{i k_{z}^{(-n)}\left(z-z_{0}\right)} e^{-i \Omega\left(t-\frac{\partial k_{z}^{(-n)}}{\partial \omega_{0}}\left(z-z_{0}\right)\right)}\left|\mathbf{A}^{(-n)}\right\rangle\left\langle\tilde{\mathbf{A}}^{(-n)} \mid \mathbf{S}_{0}\right\rangle
\end{aligned}
$$

from where, the dyadic Green function $\mathfrak{G}$ resulting from Eq. (28) can be expressed as

$$
\begin{aligned}
\mathfrak{G}\left(z, z_{0}, t\right) & =\sum_{(+n)} \theta\left(z-z_{0}\right) e^{i k_{z}^{(+n)}\left(z-z_{0}\right)} \tilde{\delta}\left(t-\frac{\partial k_{z}^{(+n)}}{\partial \omega_{0}}\left(z-z_{0}\right)\right)\left|\mathbf{A}^{(+n)}\right\rangle\left\langle\tilde{\mathbf{A}}^{(+n)}\right| \\
& -\sum_{(-n)} \theta\left(z_{0}-z\right) e^{i k_{z}^{(-n)}\left(z-z_{0}\right)} \tilde{\delta}\left(t-\frac{\partial k_{z}^{(-n)}}{\partial \omega_{0}}\left(z-z_{0}\right)\right)\left|\mathbf{A}^{(-n)}\right\rangle\left\langle\tilde{\mathbf{A}}^{(-n)}\right|,
\end{aligned}
$$

where the function $\tilde{\delta}(t)$ must be physically understood as an approximation of the Dirac delta function ", concentrated on the interval $-2 \pi / \Delta \omega \lesssim t \lesssim 2 \pi / \Delta \omega$.

Note that the obtained expression for the dyadic Green function (34) can be also formally written as

$$
\mathfrak{G}\left(z, z_{0}, t\right)=e^{\left(z-z_{0}\right)\left(i \mathfrak{K}\left(\omega_{0}, \nabla_{\mathfrak{t}}\right)-\frac{\partial \mathfrak{K}\left(\omega_{0}, \nabla_{\mathrm{t}}\right)}{\partial \omega_{0}} \frac{\partial}{\partial t}\right)} \cdot \tilde{\delta}(t)\left(\theta\left(z-z_{0}\right) \mathfrak{P}^{+}-\theta\left(z_{0}-z\right) \mathfrak{P}^{-}\right)
$$

where

$$
\mathfrak{P}^{ \pm}=\sum_{( \pm n)}\left|\mathbf{A}^{( \pm n)}\right\rangle\left\langle\tilde{\mathbf{A}}^{( \pm n)}\right|
$$

are the projection operators that separate the waves with positive and negative group delay factors. In fact, such a general form of this result could be also obtained directly by considering the solution of the homogeneous vector telegrapher's equation represented by Eq. (25). Therefore, Eq. (35) with a suitable pair of projection operators $\mathfrak{P}^{+}$and $\mathfrak{P}^{-}$can be considered as the Green dyadic solution of Eq. (28) even when the operators $\mathfrak{K}\left(\omega_{0}, \nabla_{\mathrm{t}}\right)$ and $\partial \mathfrak{K}\left(\omega_{0}, \nabla_{\mathrm{t}}\right) / \partial \omega_{0}$ do not commute.

\section{NUMERICAL RESULTS}

As a numerical example we consider propagation of a Gaussian wave packet through a uniaxial reciprocal bianisotropic medium with the transverse permittivity $\overline{\bar{\varepsilon}}_{\mathrm{t}}=\varepsilon_{\mathrm{t}} \overline{\overline{\mathrm{I}}}_{\mathrm{t}}$, the axial permittivity $\varepsilon_{\mathrm{n}}$, the transverse permeability $\overline{\bar{\mu}}_{\mathrm{t}}=\mu_{\mathrm{t}} \overline{\overline{\mathrm{I}}}_{\mathrm{t}}$, and the axial permeability $\mu_{\mathrm{n}}$. The electro-magnetic coupling dyadic $\overline{\bar{\xi}}=-\overline{\bar{\zeta}}^{T}$ is such that $\overline{\bar{\xi}}_{\mathrm{t}}=\xi_{\mathrm{t}} \hat{\mathbf{z}} \times \overline{\overline{\mathrm{I}}}_{\mathrm{t}}$

\footnotetext{
${ }^{\Phi}$ Mathematically speaking, this function is $\tilde{\delta}(t)=(2 \pi)^{-1} \int_{-\Delta \omega / 2}^{+\Delta \omega / 2} e^{-i \Omega t} d \Omega=(2 \pi)^{-1} \Delta \omega \operatorname{sinc}(t \Delta \omega / 2)$.
} 
and $\xi_{\mathrm{n}}=0$. In this case, the propagating electromagnetic waves split into two polarizations: TM polarization with $H_{z}=0$ and TE polarization with $E_{z}=0$. Considering, for example, the waves of the TM polarization propagating in the $x z$ plane, we can recast Eq. (24) in the basis of such waves with the following substitutions

$$
\mathbf{F}=\left(\begin{array}{c}
E_{\mathrm{m}, x} \\
H_{\mathrm{m}, y}
\end{array}\right), \quad \mathfrak{K}=-\omega \mathfrak{J} \cdot \tilde{\mathfrak{M}}_{\omega}, \quad \tilde{\mathfrak{M}}_{\omega}=\left(\begin{array}{cc}
\varepsilon_{\mathrm{t}} & -\xi_{\mathrm{t}} \\
\xi_{\mathrm{t}} & \mu_{\mathrm{t}}
\end{array}\right)+\frac{1}{\omega^{2}}\left(\begin{array}{cc}
0 & 0 \\
0 & \partial_{x} \varepsilon_{\mathrm{n}}^{-1} \partial_{x}
\end{array}\right), \quad \mathfrak{J}=\left(\begin{array}{cc}
0 & -1 \\
-1 & 0
\end{array}\right)
$$

where $\partial_{x}=\partial / \partial x$.

In the case when the material parameters are not uniform (e.g. because they depend on the local temperature $T$ which is not uniform), the solution of such recast Eq. (24) can be attempted numerically. We have implemented symbolic calculation code that allows one to evaluate $\mathbf{F}(z)$ by numerical integration of Eq. (24) (with 4th order Runge-Kutta method), from the initial condition at $z=0$ to an arbitrary point $z=z_{0}$. The initial field at $z=0$ is given in a form of a polynomial function in $x$ and $t$. When $z$ increases, the evolution of $\mathbf{F}$ in $x$ and $t$ is reflected in the change of the coefficients of these polynomials. The numerical integration routine also calculates the local temperature [Eq. (5)], which may in turn affect the values of the material parameters.
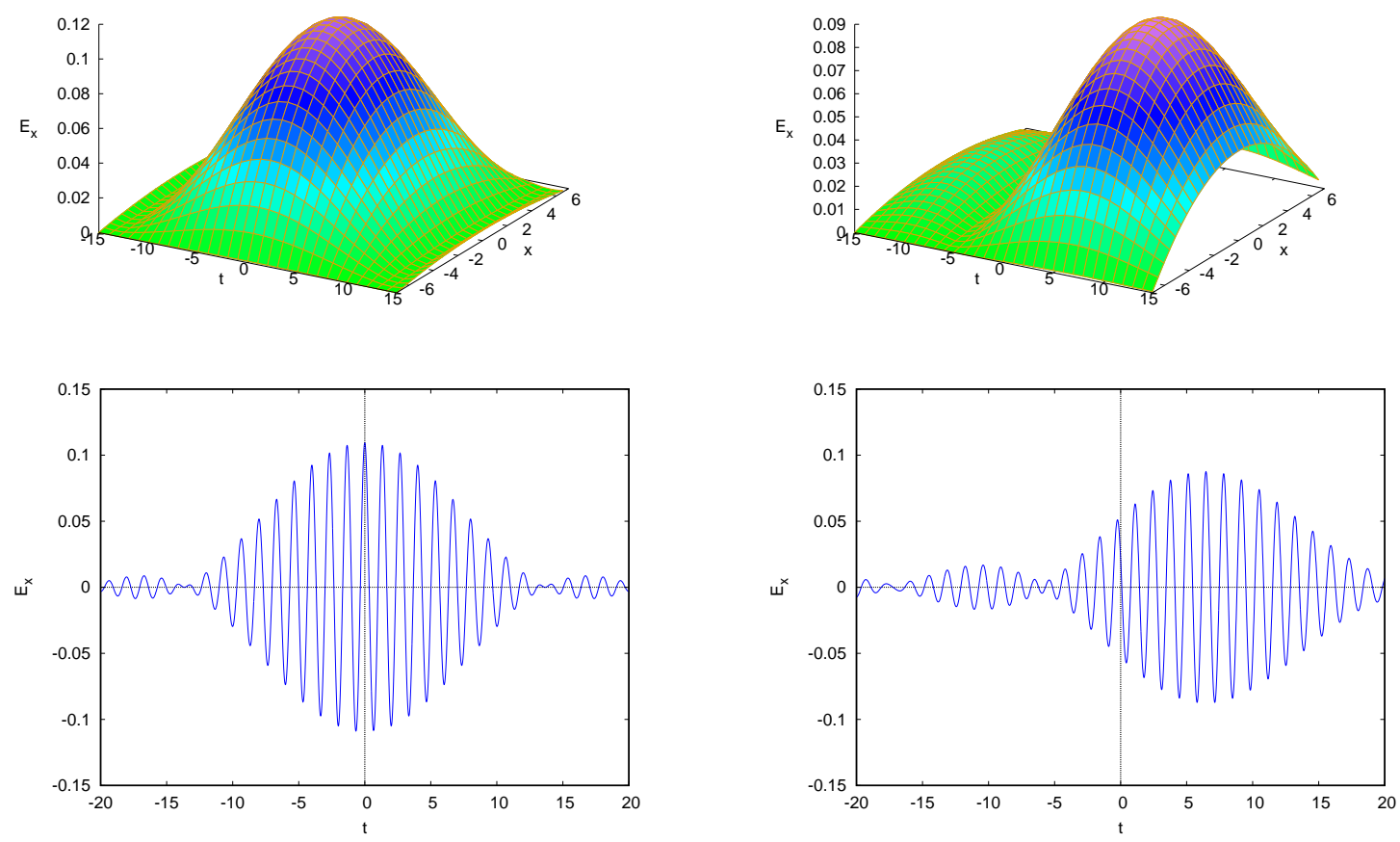

Figure 1. Top: Envelope profiles (in $x$ and $t$ ) of a pulse propagating through a bianisotropic material with Lorentzdispersive parameters (electric field, arbitrary units) at $z=0$ (left) and $z=100 \Delta z$ (right). Bottom: Same as above at $x=0$ with fast oscillations included. The parameters are $\omega_{0} / \omega_{\mathrm{p}}=4.7, \omega_{\mathrm{res}} / \omega_{\mathrm{p}}=5, \gamma / \omega_{\mathrm{p}}=0.1, \chi=0.3, \Delta z=0.1 c / \omega_{0}$. The units of $x$ and $t$ are such that $c=1 / \sqrt{\varepsilon_{0} \mu_{0}}=1$. 


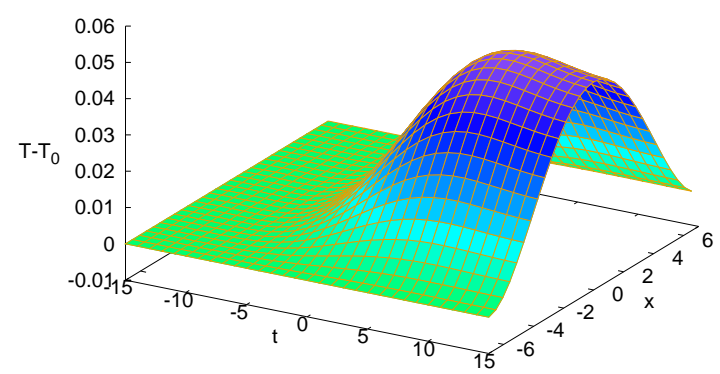

Figure 2. Local temperature profile (in $x$ and $t$ ) of a pulse propagating through a bianisotropic material with Lorentzdispersive parameters (arbitrary units) at $z=50 \Delta z$ and $c_{T}=1$. The other parameters are the same as in Fig. 1.

In the following example, we consider a bianisotropic material with Lorentz-type dispersion in $\varepsilon_{\mathrm{t}}$ and $\xi_{\mathrm{t}}$ :

$$
\varepsilon_{\mathrm{t}}(\omega)=\varepsilon_{0}\left(1+\frac{\omega_{\mathrm{p}}^{2}}{\omega_{\text {res }}^{2}-\omega^{2}-i \gamma \omega}\right), \quad \xi_{\mathrm{t}}(\omega)=\frac{i \sqrt{\varepsilon_{0} \mu_{0}} \chi \omega_{\mathrm{p}}^{2}}{\omega_{\text {res }}^{2}-\omega^{2}-i \gamma \omega},
$$

and $\varepsilon_{\mathrm{n}}=\varepsilon_{0}, \mu_{\mathrm{t}}=\mu_{\mathrm{n}}=\mu_{0}$. The characteristic evolution of the envelope of an (approximately) Gaussian wave packet is shown in Fig. 1. During the propagation the pulse amplitude is decreased due to the loss and its shape is distorted due to the dispersion. Fig. 2 shows the associated $t x$-profile of the local temperature increase during the propagation of the pulse.

\section{CONCLUSION}

In this article, we have developed a theoretical framework for studying propagation of quasi-monochromatic signals through dispersive, dissipative, bianisotropic media with weak and slow nonlinearity. By representing the time-dependent EM fields associated with such processes as products of the slowly varying complex amplitude (SVCA) and the quickly oscillating carrier we have formulated a system of equations for the SVCAs of the EM fields in such media. In the linear case, these equations resemble the vector transmission line equations, which can be solved analytically with the Green function approach in the linear uniform case, or numerically in other cases. This framework has been used to model propagation of wave packets through a material whose parameters may depend on the local temperature, which in turn is affected by the passing electromagnetic radiation. The developed framework can be used for investigating the propagation of modulated EM waves through waveguiding systems that include bianisotropic metamaterial components and also in radiative heat transfer-related problems involving such media.

\section{ACKNOWLEDGMENTS}

S. I. Maslovski acknowledges financial support from Fundação para a Ciência e a Tecnologia (FCT), Portugal, under Investigador FCT (2012) grant (Ref. IF/01740/2012/CP0166/CT0002). 


\section{REFERENCES}

[1] S.M. Rytov, Y.A. Kravtsov, and V.I. Tatarskii, Principles of Statistical Radiophysics 249 (Springer, 1989).

[2] Z.M. Zhang, Nano/Microscale Heat Transfer (McGraw-Hill, New York, 2007).

[3] S.I. Maslovski, C.R. Simovski, and S.A. Tretyakov, New J. Phys. 18, 013034 (2016).

[4] C. Simovski, S. Maslovski, I. Nefedov, and S. Tretyakov, Opt. Express 2114988 (2013).

[5] S.I. Maslovski, C.R. Simovski, and S.A. Tretyakov, Phys. Rev. B 87, 155124 (2013).

[6] H. Mariji and S.I. Maslovski, https://arxiv.org/abs/1802.05899. 